\title{
Topology Optimal Design of Wind Turbine Blades Considering Aerodynamic Load
}

\author{
Quan WANG, Xing HONG ${ }^{a,{ }^{*}}$, Zheng-Zheng QIN, Jun WANG, Jin-Feng SUN \\ and Jun REN
}

\author{
School of Mechanical Engineering, Hubei University of Technology, Wuhan, 430068, China \\ a1352614342@qq.com \\ ${ }^{*}$ Corresponding author
}

\begin{abstract}
Keywords: Wind Turbine Blade, Conceptual Design, Grid Technology, Aerodynamic Loads, Topology Structure.
\end{abstract}

\begin{abstract}
For the conceptual design problem of the complex blade aerodynamic structure, the parametric finite element model of the wind turbine blade was established using 3D blade integrated expressed functions. The blade section topology structure technology which can solve the whole hexahedral grid problem for the solid blade was presented. Based on the modified blade element momentum theory, the aerodynamics were loaded to the blade surface. A procedure combining MATLAB and APDL to put the pressure distribution on the blade finite element model is developed. The solid blade model which the objective function was the minimum weight was optimized. Compared with the traditional blade structure, the location of main beams with no web for the optimized blade which exhibit asymmetric property were near to blade leading edge. Moreover, the trailing edge of the newly blade had reinforced structure. The newly topology structure of the blades had important guiding significance for the sizing optimization of wind turbine blades.
\end{abstract}

\section{Introduction}

With the wind power more and more large, the blade size is also longer and longer. How to improve the effectiveness of the structure of the blade is particularly important. An effective method is to ensure the quality of the blade structure under the premise of how to reduce the quality of the blade. Researchers at home and abroad have done a lot of research work on how to improve the performance of wind turbine blades and reduce their quality. X. D. Wang [1] et al. proposed a multidisciplinary optimization design model of the blade of the wind turbine. The optimal output of a 5MW wind turbine blade was designed with the energy cost of the wind turbine as the objective function, which reduced the energy cost per unit output of the wind turbine by $2.6 \%$. The effectiveness of the method has been verified by comparing the load distribution as well as the power characteristics of the optimized blade and the original blade. Q. Wang [2] et al. proposed a new one-way blade fluid-solid coupling method, and applied the method to the parametric composite finite element model of wind turbine blade. The thickness of the blade and the position of the main beam were designed and optimized. The optimization results show that the blade quality is reduced by $7.14 \%$ under the condition of failure criterion and displacement. In recent years, the application of topology to the design and optimization of blade structure of wind turbine has gradually become a hot research field. Some scholars abroad have also carried out relevant research. In 2010, E. Lindgaard and E. Lund [3] of the University of Elborg in Denmark proposed a topological optimization method that takes into account the structural stability of the composites. The laying angle can be used as a design variable and applied to the wind turbine blade main girder optimization of Linear and Nonlinear Buckling Stability. The above results are used to evaluate the merits of the optimization results by eigenvalues and sensitivity analysis. In 2012, J. P. Blasques [4] combined the structural topology with beam section mechanical properties and regarded the minimum compliance as the objective function, the material volume ratio for the design variable, the blade section weight and the center of gravity as the constraint function to design the composite beam cross section Material topology optimization. Then he verified the effectiveness of the method by taking the blade cross section of the composite wind turbine as an example. In 2013, N. Buckney [5] et al. analyzed the blade structure effectiveness of a 3MW wind 
turbine blade according to the effectiveness of its material. It indicated that the topological optimized blade section had better structural effectiveness. The above studies have made fruitful research on the structure of the pavement, the strength characteristics, the nonlinear stability and the structural validity. However, in the design of the most primitive structural topology of the blade, the aerodynamic load characteristics of the blade were not taken into account and it is difficult to make breakthrough progress. As the wind turbine blades are influenced by the aerodynamic, centrifugal force and inertial force and other alternating load role. It makes the wind turbine blades to ensure a high aerodynamic performance, but also to meet a certain stiffness, strength, vibration characteristics and stability requirements. And this form of force will directly affect the structure of the blade topology.

Therefore, this paper will carry out aerodynamic load under the action of wind turbine blade topology optimization problem. A $2 \mathrm{MW}$ new type of wind turbine blade is selected. Based on the integrated expression of three - dimensional blade complex surface, the data transfer mechanism is established by MATLAB and APDL language program, and the finite element model of parametric blade 3D solid element is established. A new geometric topology is proposed for the complex shape of the blade section, which can realize the full hexahedral element of the blade. Considering the aerodynamic load on the aerodynamic surface of the blade, the finite element model of the blade topology optimization is established with the minimum weight (minimum volume) as the objective function, and the topology optimization results are given.

\section{Blade Geometry and Gird Technology}

In this study, a new $2 \mathrm{MW}$ wind turbine blade was selected as the topological design object. The blade aerodynamic shape is designed by the CQU-A airfoil series with light-weight and high performance as the objective function [6]. The blade chord length, twist angle and maximum relative thickness change along the blade length direction as shown in Fig.1. Based on the integrated expression of three-dimensional blade complex surface [6], the airfoil is coupled into the integrated expression, and the program is transformed into a spatial three-dimensional data point. Besides, the macro file is compiled with APDL language and the data transfer mechanism is formed to establish the blade parameter finite element model, as shown in Fig.2.

This paper presents a blade topological topology technique. As shown in Fig.3 (a), the complex blade section is divided into six geometric topological regions, and each topological region can be divided into hexahedral meshes. Finally, the whole blade can achieve hexahedral mesh. Select a blade airfoil, the hexagonal element finite element model is shown in Fig.3 (b). It can be seen that the hexahedral element can capture the topology better and its aerodynamic surface is smoother than the tetrahedral element. So it is conducive for aerodynamic loading.

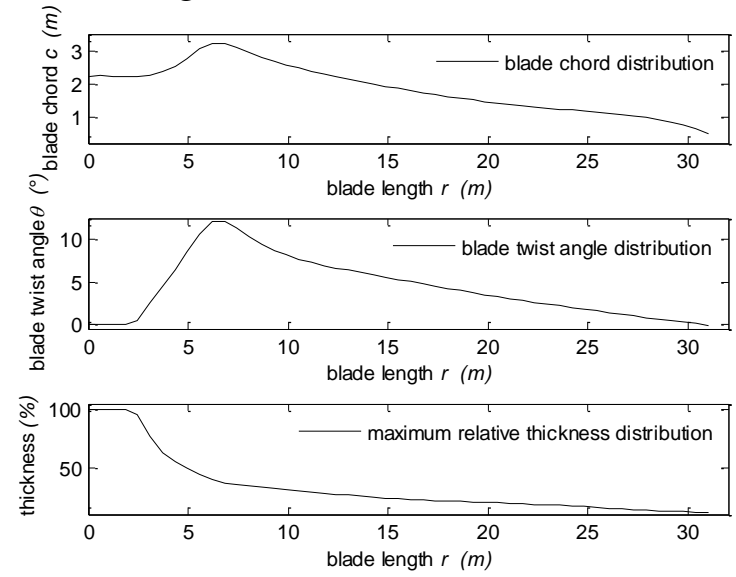

Fig. 1 Blade Chord Length, Twist Angle and Thickness Distribution 


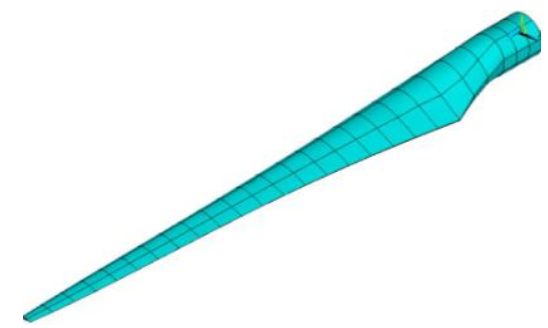

Fig. 2 Three-dimensional Geometric Model of Wind Turbine Blade

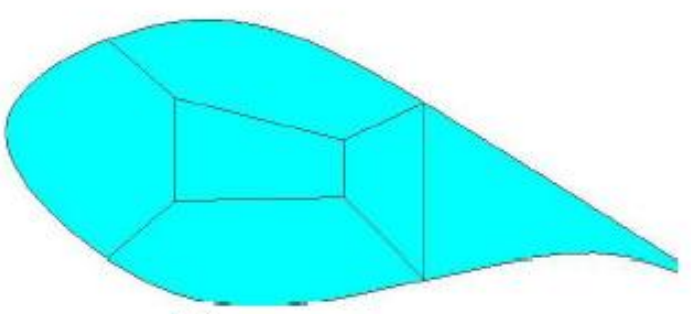

(a)

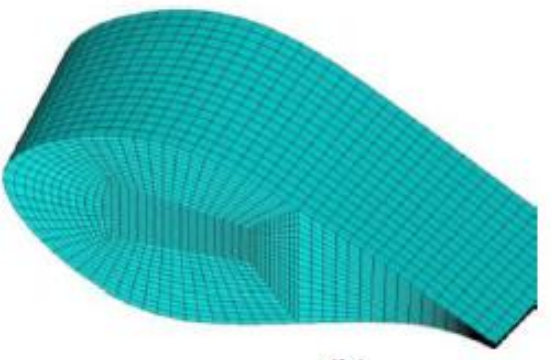

(b)

Fig. 3 Blade Hexahedral Mesh Topolog

\section{Aerodynamic Load and Boundary Conditions}

Wind turbine blade is always influenced by the aerodynamic, gravity and inertia force load coupling at the same time. In the past, the blade structure topology design usually converts the aerodynamic load into concentrated bending moment and torque to load, while ignoring the true blade surface pressure distribution characteristics. Therefore, this study considers the blade surface pressure distribution, for the initial topology optimization design of wind turbine blade. Combined with the aerodynamic data calculated by RFOIL software and the theory of momentum leaf [7], the pressure distribution data of each airfoil section under normal operating conditions can be obtained. The aerodynamic pressure acting on the blade can be obtained according to equation (1):

$$
\mathrm{p}=\frac{1}{2} \rho v_{r e l} C_{p}+p_{\infty}
$$

Where $\rho$ is the air density of $1.205 \mathrm{~kg} / \mathrm{m} 3, C_{p}$ is the pressure coefficient, the Attack Angle can be calculated by using RFOIL software, $P_{\infty}$ is the standard atmospheric pressure, $v_{\text {rel }}$ is the relative acting on the blade, based on the theory of momentum leaf element can be obtained relative velocity, according to equation (2):

$$
v_{\mathrm{rel}}=\sqrt{v_{x}^{2}+v_{y}^{2}}=\sqrt{(1-a)^{2} v_{0}^{2}+(1+b)^{2}(\omega r)^{2}}
$$

Where, $v_{0}$ —wind speed,

$\omega$-Wind wheel rotation angular velocity,

$\mathrm{r}$ - the position along the blade,

$\mathrm{a}, \mathrm{b}$ - axial induction factor and circumferential induction factor,

$\mathrm{v}_{\mathrm{x}}, \mathrm{v}_{\mathrm{y}}$ - the velocity component of the incoming wind speed in the $\mathrm{x}, \mathrm{y}$ direction.

While the axial induction factor a and the circumferential induction factor $\mathrm{b}$ can be expressed as:

$$
a=\frac{2+Y_{1}-\sqrt{4 Y_{1}(1-F)+Y_{1}^{2}}}{2\left(1+F Y_{1}\right)}
$$




$$
b=\frac{1}{(1-a F) Y_{2} /(1-a)-1}
$$

Where, $Y_{1}=4 F \sin ^{2} \phi /\left(\sigma C_{\mathrm{n}} F_{1}\right), Y_{2}=4 F \sin \phi \cos \phi /\left(\sigma C_{t} F_{1}\right), F=\frac{2}{\pi} \cos ^{-1}\left[\exp \left(-\frac{B(R-r)}{2 r \sin \phi}\right)\right]$,

$$
\begin{aligned}
& \phi=\arctan \frac{(1-a) v_{0}}{(1+b) \omega r}, C_{n}=C_{l} \cos \phi+C_{d} \sin \phi, C_{t}=C_{l} \sin \phi-C_{d} \cos \phi, \\
& F_{1}=\frac{2}{\pi} \cos ^{-1}\left[\exp \left(-g \frac{B(R-r)}{2 r \sin \phi}\right)\right], \sigma=B c /(2 \pi r), g=\exp [-0.125(B \lambda-21)]+0.1,
\end{aligned}
$$

$\lambda=w R / v_{0}, \mathrm{R}$ is the length of the blade,

The programming procedure is iteratively solved for $a$ and $b$, into the equation (2) can obtain the relative velocity of each blade section, and then according to equation (1) to calculate the pressure distribution on each blade. Figure 4 shows a comparison of the fitting pressure distribution versus the discrete pressure distribution at $25 \%$ from the blade (Wind speed $12.5 \mathrm{~m} / \mathrm{s}$, rated speed of $20 \mathrm{r} / \mathrm{min}$ ).

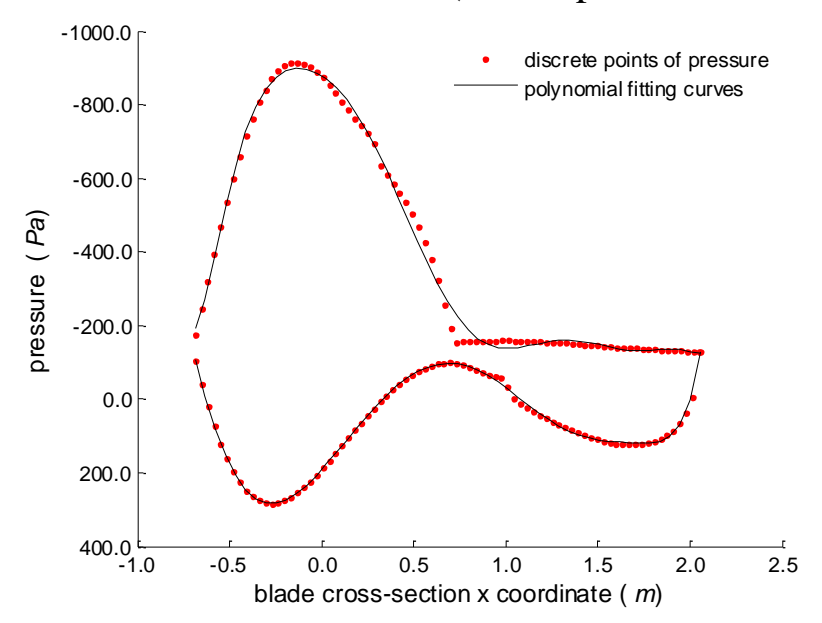

Fig. 4 Leaf Section Pressure Distribution at the Position $r / R=0.25$

The material properties are quasi-isotropic materials with a Young's modulus of $44 \mathrm{GPa}$, a shear modulus of $17.3 \mathrm{GPa}$ and a Poisson's ratio of 0.27 [5]. Although the characteristics of the wind turbine blade material are anisotropic (the blade is made of composite material), and for the solid element can not be anisotropic material to simulate. But for the initial design of the blade topology, the use of isotropic structural unit also has a guiding significance [5].

\section{Topology Optimization and Result Analysis}

The purpose of topology optimization is to find a reasonable material density distribution and an optimal force transfer path in the design area. This paper is based on SIMP material interpolation model, the discretization problem is transformed into continuous optimization problem. The optimization criterion is based on MMA algorithm. Under the action of aerodynamic load, the mathematical model of three-dimensional blade structure topology optimization is established by using the lightweight (minimum volume) as the objective function under the stress and deformation of tip as constraint conditions,

Objective function: $\mathrm{f}(\mathrm{x})=\min (\mathrm{V})$,

Restrictions: $\sigma_{\mathrm{m}} \leq 175 \mathrm{MPa}$,

$$
\delta_{\text {tip }} \leq 6 m,
$$


Where $\sigma_{\mathrm{m}}$ is the von Mises stress, $\delta_{\mathrm{tip}}$ is the direction of waving beat tip deformation.

The topology optimization results are shown in Fig.5, the main girders of the vanes extend from the root to the tip of the blade in the direction of the spreading, and the girders of the main girder are concentrated along the leading edge of the blade and the maximum thickness.

In order to see the details of the topology, the blade sections were studied along the orientation of about $25 \%$ and $80 \%$, respectively (as shown in Figures 6 and 7). It can be seen from the figure, the width of the main beam in the windward surface (pressure surface) is wider than that on the leeward side (suction surface). As the main beam extends to the tip, the main beam of the blade covers the whole windward surface and the web structure appears.

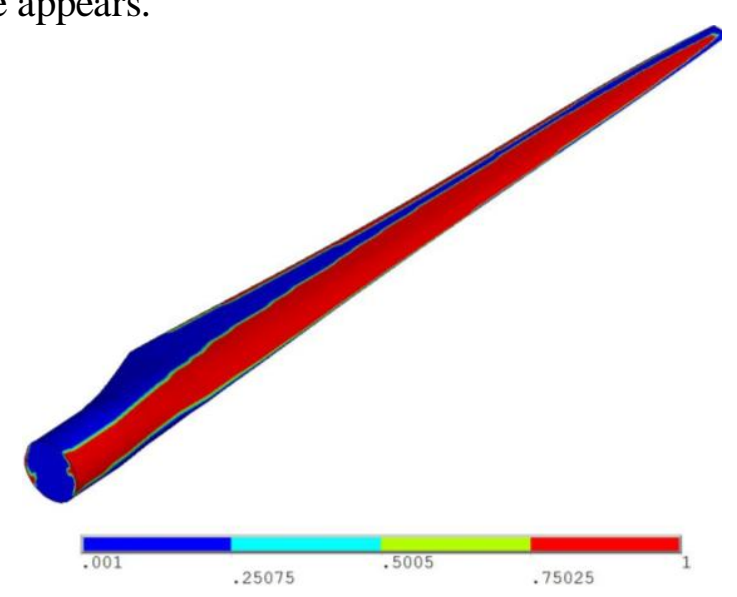

Fig. 5 Overall Blade Topology Optimization Results
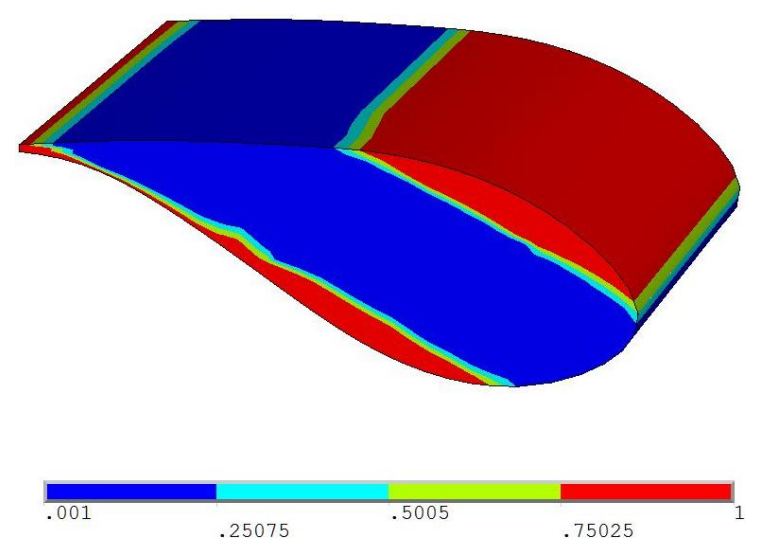

Fig. 6 Along the Blade Extension of About 25\% of the Topological Optimization Results

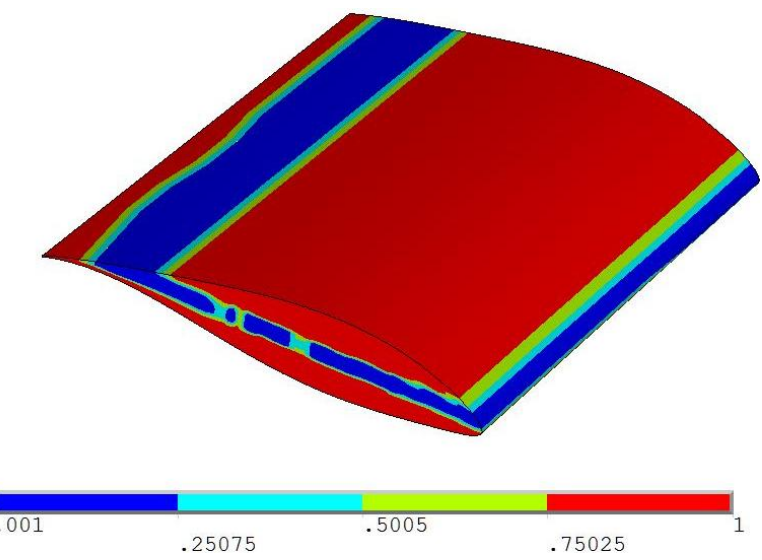

Fig. 7 Along the Blade Extension of About $80 \%$ of the Topological Optimization Results 


\section{Summary}

For the complex three - dimensional blade solid model, based on the integrated expression equation of blade and MATLAB and APDL language programming program to achieve data transfer mechanism to establish the wind turbine blade parametric finite element solid model. A new mesh topological path is proposed for the complex blade cross-sectional shape. The grid technology can realize the hexagonal mesh division of the whole blade, thus saving the computing resources and time. Considering the effect of aerodynamic load on the blade, the topology of the wind turbine blade is optimized. Optimization results show that the upper and lower airfoils of the blade are clearly visible from the blade root to the tip of the blade, mainly in the maximum thickness of the blade and the leading edge of the blade. Besides, the upper and lower girders exhibit asymmetry, mainly due to the aerodynamic load distribution Characteristics. In addition, the blade edge of the edge of the material to enhance the structural characteristics, which will undoubtedly enhance the blade anti-torsional stiffness of the ability. The follow-up work will be based on this study, based on the blade topology optimization to realize the optimization of the structure size, in order to study composite blade internal layer structure characteristics.

\section{Acknowledgment}

Fund project: National Natural Science Fund Project (51405140).

\section{References}

[1] X.D. Wang, L.C. Wang, P. Li, G.M. Gong, The shape optimization of wind turbine blades based on multidisciplinary design optimization, J. Mechanical Science and Technology for Aerospace Engineering. 05 (2012) 806-809.

[2] Q. Wang, J. Chen, et al, Structural optimization of composite wind turbine blade under aerodynamic load, J. Journal of Mechanical Engineering. 50 (2014) 114-121.

[3] E. Lindgaard, E. Lund, Nonlinear buckling optimization of composite structures, J. Computer Methods in Applied Mechanics and Engineering. 199 (2010) 2319-2330.

[4] J. P. Blasques, M. Stolpe, Multi-material topology optimization of laminated composite beam cross section, J. Composite Structures. 94 (2012) 3278-3289.

[5] N. Buckney, A. Pirrera, et al, Structural efficiency of a wind turbine blade, J. Thin-Walled Structures. 67 (2013) 144-154.

[6] J. Chen, Q, Wang. Wind turbine wing and blade optimization design theory, first ed., Science Press Co. Ltd, Beijing, 2013.

[7] W. Z. Shen, R. Mikkelsen, J. N. Sorensen, Tip loss correction for wind turbines computations, J. Wind Energy. 5 (2005) 457-475. 